\title{
Role of multi-detector computed tomography angiography in the evaluation of lower limb ischemia
}

\author{
Maged Abdelfattah Ali Algazzar ${ }^{1}$, Mohamed Salah Eldin Elzawawi ${ }^{1}$, \\ Khaled El-Sayed Alhawary ${ }^{2}$, Waleed AbdelFattah Mousa ${ }^{1}$ \\ ${ }^{1}$ Radiology department, Faculty of Medicine, Menoufia University, Menoufia, Egypt \\ ${ }^{2}$ Radiology department, National Liver Institute, Menoufia University, Menoufia, Egypt
}

\section{Email address:}

dr.maged.algazzar@gmail.com (M. A. Algazzar),melzawawi66@yahoo.com (M. S. E. Elzawawi), waleedmousa75@gmail.com (W. A. Mousa)

\section{To cite this article:}

Maged Abdelfattah Ali Algazzar, Mohamed Salah Eldin Elzawawi, Khaled El-Sayed Alhawary, Waleed AbdelFattah Mousa. Role of Multi-Detector Computed Tomography Angiography in the Evaluation of Lower Limb Ischemia. International Journal of Medical Imaging. Vol. 2, No. 5, 2014, pp. 125-130. doi: 10.11648/j.ijmi.20140205.16

\begin{abstract}
Objective: To evaluate the value of MDCT Angiography in imaging the arterial tree of the lower limbs in peripheral vascular disease. Back-ground: Peripheral arterial disease (PAD) is defined as any pathologic process causing obstruction to blood flow in the arteries, exclusive of the coronary and cerebral vascular beds. Conventional angiography is associated with $1-2 \%$ rate of complications and requires highly skilled physician to perform it. Non-invasive imaging modalities include duplex ultrasonography, magnetic resonance angiography (MRA), and multi-detector computed tomography angiography (MDCTA). Methods: This study includes 30 patients of variable ages, complaining from peripheral arterial disease manifestations referred to the radiology department of Menoufia University hospital. All patients underwent multi-detector row CT angiography of the lower limbs arteries. The findings were then analyzed and retrospectively compared with the surgical outcome in cases of operative intervention or Color Doppler correlation. Results: 120 lesions were detected in which 12 lesions $(10 \%)$ were noted at the aortoiliac vessels, 33 lesions $(27.5 \%)$ were noted at the femoropopliteal vessels in and 75 lesions $(62.5 \%)$ were noted at the infrapopliteal vessels. No statistically significant difference between Doppler and CT angiography regarding all the anatomic segments of the arterial tree. Conclusion: Multi-detector CT angiography has demonstrated its efficacy as a promising new, fast, accurate, safe and a minimallyinvasive imaging modality in cases of trauma with suspected arterial injuries and in cases of peripheral vascular diseases for diagnosis, grading and for preoperative evaluation. It can also replace color Doppler ultrasonography in many cases.
\end{abstract}

Keywords: Peripheral Arterial Disease, MDCTA, Duplex Ultrasonography

\section{Introduction}

Peripheral arterial disease (PAD) is a common manifestation of atherosclerosis and is defined as any pathologic process causing obstruction to blood flow in the arteries, exclusive of the coronary and cerebral vascular beds. PAD affects a large segment of the adult population. Less than $20 \%$ of patients with PAD have typical symptoms of intermittent claudication, whereas another third have atypical exertional leg symptoms. ${ }^{1}$

Management strategies are governed by the severity of the disease. Imaging is necessary for planning interventions in patients with lower extremity peripheral arterial disease $(\mathrm{PAD}){ }^{2}$ Non-invasive imaging modalities, including duplex ultrasonography, magnetic resonance angiography (MRA), and multi-detector computed tomography angiography (MDCTA) are available for grading lower extremity arterial disease. Duplex ultrasonography has a high specificity of $95 \%$ and a somewhat lower sensitivity of $88 \%$ for detecting hemodynamically significant lesions ( $>50 \%$ stenosis or occlusion). ${ }^{3}$

Computed tomography angiography is increasingly attractive due to rapid technical developments. Shorter acquisition times, thinner slices, higher spatial resolution, and improvement of multi-detector computed tomographic (CT) scanners enable scanning of the whole vascular tree in a limited period with a decreasing (but still substantial) amount of contrast medium. Recent studies on CTA report sensitivity 
and specificity rates of around $98 \%$ for detecting PAD. ${ }^{4}$

\section{Methods}

This study included thirty patients who were referred to the Radiology department of Menoufia University hospital with suspected lower limb ischemia. The study was conducted in Menoufia University Hospital during the period from January 2013 to June 2014.

The patient population consists of 18 males and 12 females with age ranging from 24-75 years with a mean of 56 years.

Full history was taken from every patient with clinical examination and revision of previous available studies like duplex ultrasonography. Patient's laboratory data was initially revised with particular interest in the results of the renal function tests. All patients were asked to continue adequate simple fluid intake up to 3 hours prior to examination to ensure adequate hydration. Patients were taught how to hold breath during examination when requested, to ensure their cooperation.

Patients then underwent lower limb CT angiography with a sixteen channel multi-detector row CT scanner (Toshiba Aquilion 16 CT Scanner). Patients were positioned supine on the CT table in the "foot first" position with an 18-20 gauge catheter placed into a superficial vein within the antecubital fossa. Two scouts were acquired, anteroposterior and lateral. The examination was planned on these scouts from the level of D12 till the foot. Patients were requested to hold their breath during the first 20 seconds of the acquisition and were allowed to breathe quietly after that.

The scan technique was $120 \mathrm{kV}$ at $150 \mathrm{mAs}(300 \mathrm{~mA}$ at $0.5 \mathrm{~s}$ rotation), using $1 \mathrm{~mm} \times 16$ slices. On helical acquisition automatic $\mathrm{mA}$ modulation was used to reduce patient dose (Sure Exposure technology, Toshiba Medical Systems).

A detector configuration of $16 \times 1.0 \mathrm{~mm}$ is used, and 1.0 $\mathrm{mm}$ thick sections are reconstructed at $1 \mathrm{~mm}$ intervals. Acquisition timing for optimum opacity is achieved by using automatic bolus tracking (Sure Start technology, Toshiba Medical Systems) of a region of interest placed on an artery. The ROI is placed on the common femoral artery in the groin. The trigger level is set at 180 Hounsfield units. The study is usually performed with $150 \mathrm{cc}$ of low osmolar non-ionic contrast medium (Omnipaque 350) with a flow rate $4 \mathrm{cc} / \mathrm{sec}$. The sure start technique eliminates the problem of the table outpacing the contrast agent in the proximal vessels. In the distal vessels we overcome this problem by performing a second run from the knee to the foot.

Following completion of the scan axial $1 \mathrm{~mm}$ slices are reconstructed and automatically forwarded for 3D processing on the Vitrea workstation. Three dimensional maximum intensity projections (MIP), volume rendering (VR) and curved planer reformations .Figure $(1,2)$. Each CT angiography was interpreted and the arterial tree was always studied part by part, from the abdominal aorta up to the ankle. The criteria of analysis were as follows:

- opacification or non-opacification of the studied part ;

- $\quad$ absence or presence of significant stenosis (more than $50 \%$ ) and estimation of its length ;

- absence or presence of an occlusion and estimation of its length

- $\quad$ presence of artery wall calcifications masking, or not masking the lumen and assessment of their distribution.

The arterial tree was then divided into three segments including aortoiliac, femoropopliteal, and infrapopliteal segments to assess the segmental distribution of the disease.

No complications what so ever occurred during the multi-detector row CT angiography examination including contrast extravasation or reaction.

Color Doppler sonography examinations were done using HITACHI: EUB-7000 HV, Patients were examined in the supine position. Beginning at the aortic bifurcation, a $3.5 \mathrm{MHz}$ probe was used to examine the aorta, common, and external iliac arteries. A 7.5 $\mathrm{MHz}$ probe was sometimes used in well prepared thin patients. Both common femoral arteries, superficial femoral, polpiteal, anterior and posterior tibial arteries were examined using a $7.5 \mathrm{MHz}$ probe with the limb under examination slightly abducted and externally rotated for better scanning of the femoral artery.

Each part was examined first by B-mode for detection of atheromatous plaques and wall calcifications then by color flow imaging transversely and longitudinally to size the colored flow in the lumen with respect to the arterial wall and to detect areas of flow disturbance, increased velocity, and jets. The transducer was transverse to the arterial segment with $30^{\circ}$ angulation to the vertical plane to encode the arterial color signal.

\section{Results}

This study included 30 patients .They were 18 male and 12 female. The age of the patients ranged from 24 to 75 years old with average age of presentation 56.3 years.

Fifteen patients $(50 \%)$ were hypertensive, ten were diabetics $(10 \%)$, seventeen $(56.67 \%)$ where smokers with seven of them $(23.33 \%)$ chronic heavy smokers.

Twelve $(40 \%)$ of them complained of intermittent claudication, seven $(23.33 \%)$ complained of rest pain, five (16.67\%) with acute lower limb pain and three of them presented with gangrene (10\%). Figure (3).

The arterial system was divided into 21 anatomical part :

Each and every anatomical part of the arterial tree was assigned a grade for the disease extent. These grades were given for all arterial parts as evaluated by multi-detector row CT angiography and color Doppler ultrasonography.

Multi-detector row CT angiography was used to examine a total of 624 arterial segments showing 120 lesions. Out of the detected 120 lesions: 12 lesions $(10 \%)$ were noted at the 
aortoiliac vessels, 33 lesions $(27.5 \%)$ were noted at the femoropopliteal vessels in and 75 lesions (62.5\%) were noted at the infrapopliteal vessels. Table 1

The PAD showed multisegmental distribution: affecting 1 segment in 9 patients (30\%), two segments in 11 patients $(36.67 \%)$ and three segments in 10 patients $(33.33 \%)$. Table 2.

Table 1. Distribution pattern of PAD detected by CTA in our study

\begin{tabular}{lllll}
\hline & Mild & $\begin{array}{l}\text { Moderate } \\
\text { to Severe }\end{array}$ & Occluded & Total \\
\hline -Aorto iliac & 4 & 4 & 4 & $12(10 \%)$ \\
$\begin{array}{l}\text {-Femoro -popliteal } \\
\begin{array}{l}\text {-Crural vessels } \\
\text { (infra popliteal }\end{array}\end{array}$ & 6 & 7 & 20 & $33(27.5 \%)$ \\
arteries) & 14 & 22 & 39 & $75(62.5 \%)$ \\
\hline
\end{tabular}

Table 2. Segmental distribution of PAD as detected by CTA in our study

\begin{tabular}{lll}
\hline & $\%$ & $\mathbf{N}=\mathbf{3 0}$ \\
\hline -One segment & $30 \%$ & 9 patients \\
-Two segments & $36.67 \%$ & 11 patients \\
-Three segments & $33.33 \%$ & 10 patients \\
\hline
\end{tabular}

No statistically significant difference between Doppler and CT angiography regarding all the examined lower limb arterial tree segments except for the internal iliac artery which was not examined by Doppler sonogrpahy.

\section{Discussion}

Various imaging techniques are used in the diagnosis of lower extremity arterial occlusive disease (LEOD). The usual are duplex scan ultrasonography and conventional angiography. The gold standard conventional angiography is responsible for complications in 1 to $2 \%$ of patients. For this reason non invasive techniques have been recently developed. $^{5}$

CT angiography (CTA) of the lower extremities has evolved into a robust noninvasive angiographic technique with the advent of 16 and 64 multidetector computed tomographic systems and advances in system design. CTA has displaced conventional catheter arteriography in a large range of applications and is predominantly used in the evaluation of atherosclerotic peripheral arterial occlusive disease in symptomatic patients who are candidates for intervention. Other disease entities including atheroembolism and thromboembolism, aneurysmal disease, and arteritides including Buerger disease and Takayasu arteritis can be precisely evaluated by CTA. ${ }^{6}$

Fleischmann et al ${ }^{7}$ described the imaging of the entire arterial supply of the lower extremities using sixteen channel multi-detector row $\mathrm{CT}$ in a single helical acquisition using a detector configuration of $16 \times 1.25 \mathrm{~mm}$ or $16 \mathrm{x} 1.5 \mathrm{~mm}$ with 1.25-2-mm-thick sections reconstructed at $0.8-1-\mathrm{mm}$ intervals.

Our study also used a similar protocol. We used a detector configuration of $16 \times 1.0 \mathrm{~mm}$ and $1.0 \mathrm{~mm}$ thick sections are reconstructed at $1-\mathrm{mm}$ intervals .Our gantry rotation period was $0.5 \mathrm{sec}$, while his was $0.75 \mathrm{sec}$.
In multi-detector row CT angiography we are dealing with a huge number of axial images (800-1000 image). Rubin et al ${ }^{8}$, stated that this "data explosion" was considered to be the greatest challenge of the new multidetector row CT scanners. Using 3D techniques might help to solve this problem. Yet, reviewing the axial images by the radiologist is mandatory.

The MIP algorithm, one of the most commonly used formats, has the capability to reveal the entire vascular tree in one image but, at present, has limitations. Bones and dense calcifications obscure the true lumen of the arteries and must be erased from the projections produced. ${ }^{9}$

There are no current studies comparing color Doppler sonography and MDCT angiography. Willmann et al ${ }^{10}$ made a prospective blinded comparative study between MDCT angiography, DSA and color Doppler sonography in evaluation of peripheral arterial bypass grafts. That study included 85 arterial bypass grafts. There were no significant differences in sensitivity and specificity between MDCT angiography and CCD in detection of hemodynamically significant lesions

This was similar to our results as we examined 21 arterial segments with no statistically significant difference between Doppler and CT angiography regarding all the anatomic segments

Ozkan et al ${ }^{11}$ examined the segmental distribution of atherosclerosis in 626 symptomatic patients with peripheral arterial disease. Peripheral arterial disease involved one segment in $36 \%$ of the patients, two segments in $42 \%$ of the patients and three or more segments in $33.33 \%$ of the patients. He concluded that PAD was multisegmental in most of the cases of the study group

This was similar to our results where PAD involved one segment in $30 \%$ of the patients, two segments in $36.66 \%$ of the patients and three segments in $33.33 \%$ of the patients. Figure $(4,5)$.

\section{Conclusion}

MDCT angiography is a fast, accurate, safe and a minimally-invasive imaging modality which may be used in cases of trauma with suspected arterial injuries, in cases of peripheral vascular diseases for diagnosis, grading and for preoperative assessment of lower limb arterial disease.

The limiting factors that prevent the widespread usage of MDCT angiography are the limited number of multidetector row CT machines and the limited experienced stuff that can perform such a recent examination. Interpretation of the images by a radiologist with experience in vascular imaging combined with experience in multi-detector row CT imaging is mandatory.

Doppler ultrasonograpy is a reliable non-invasive method of investigating the lower limb arterial system. It has an advantage over MDCT angiography that it provides us with hemodynamic data proximal, distal and at the site of obstruction. The limiting factor for color Doppler sonography imaging is that this examination is totally 
operator dependent. It requires highly trained personnel which is not always available. It also lacks the arterial imaging capabilities of MDCT angiography that surgeons need for preoperative planning. It can only document a small arterial segment in each image. This leads us to the conclusion that MDCT angiography may replace color Doppler sonography in many cases.

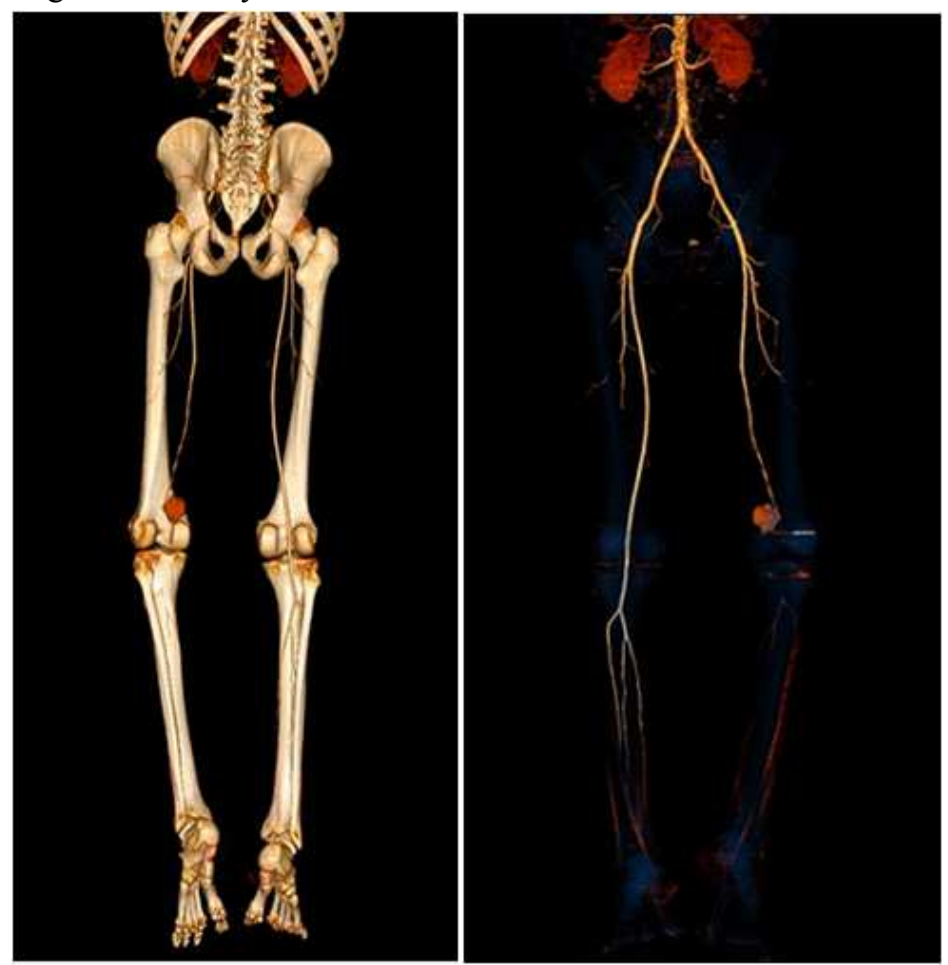

Figure (1). Traumatic injury of the mid left popliteal artery. Volume rendered image (posterior view and anterior view) showing the active contrast extravasation and hematoma formation with absent distal opacification of the popliteal artery and faint opacification of the below knee arteries.

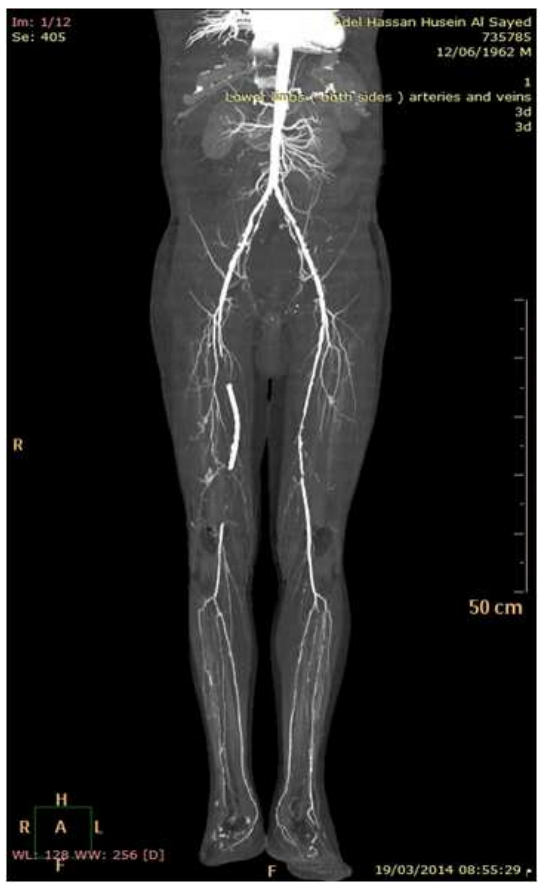

Figure (2). Acute right lower limb ischemia on top of chronic bilateral lower limb ischemia. MIP image showing occlusion of the mid right SFA , and absent flow through the SFA stent. 

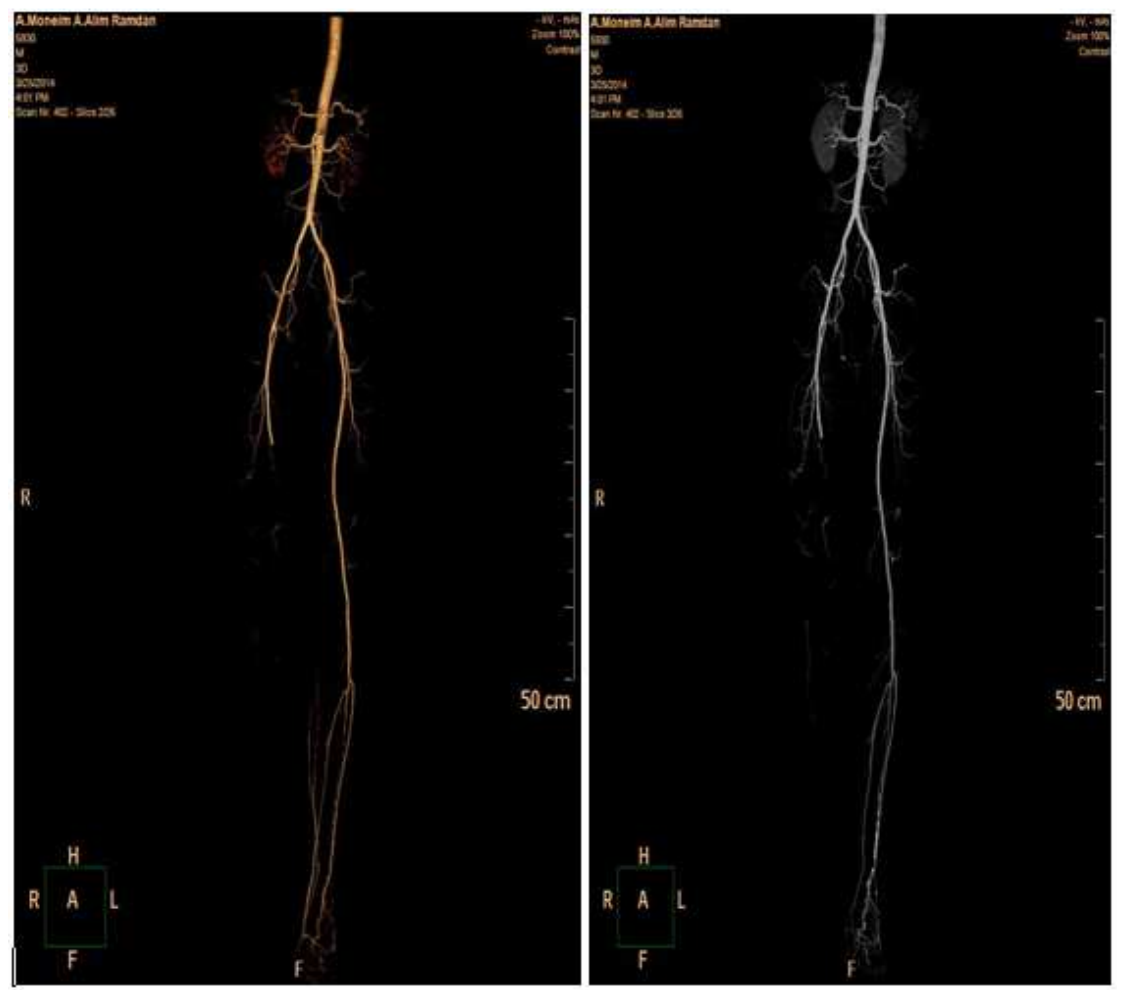

Figure (3). Acute Ischemia :Volume rendered image and maximum intensity projection showing occlusion of the right distal superficial femoral artery with weak collateral filling through some genicular collaterals and absent distal flow .

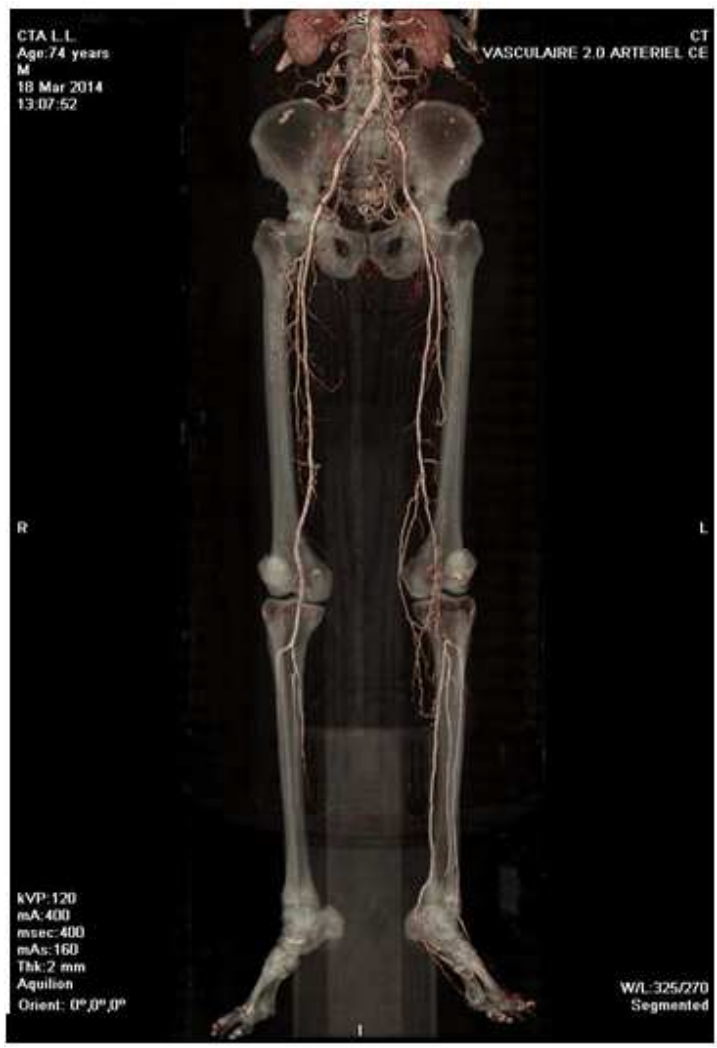

Figure (4). Acute right lower limb ischemia on top of chronic bilateral lower limb ischemia. Volume rendered image reveals left lower limb chronic ischemia with occlusion of the popliteal artery with genicular anastmosis and reconstitution of the infrageniculate vessels. The right lower limb infrageniculate vessels are seen occluded at the mid tibial level with no flow could be detected distally. 


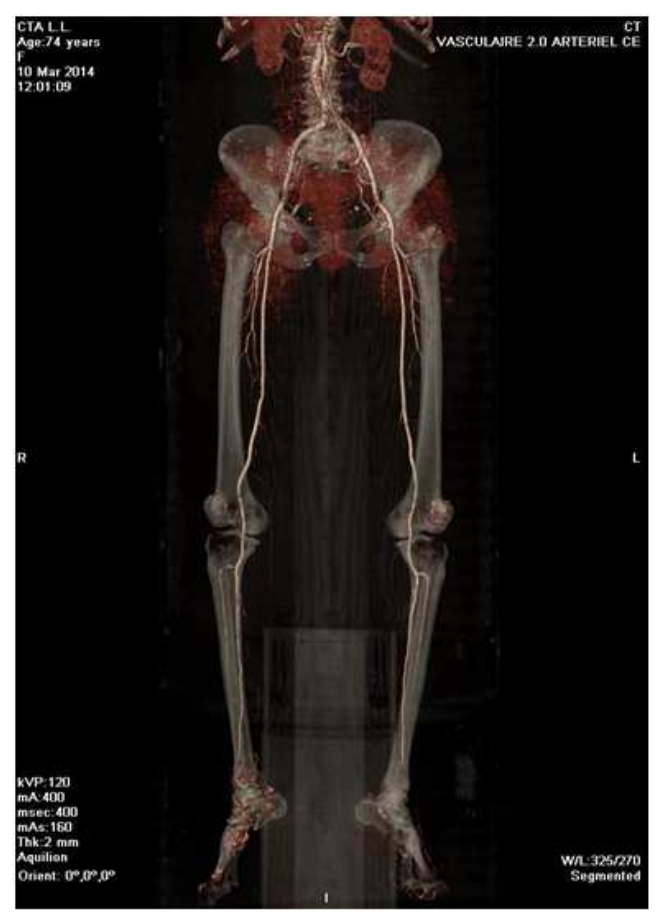

Figure (5). Chronic bilateral lower limb ischemia with sparing of the peroneal artery. Volume rendered image reveals occlusion of both posterior tibial arteries with bilaterally weak opacification and attenuation of the anterior tibial arteries. The peroneal artery is spared bilaterally.

\section{References}

[1] Kasapis C , Gurm HS: Current Approach to the Diagnosis and Treatment of Femoral Popliteal Arterial Disease. A Systematic Review.Curr Cardiol Rev 2009; 5(4): 296-311.

[2] Collins R, Burch J, Cranny G , Westwood M: Duplex ultrasonography, magnetic resonance angiography, and computed tomography angiography for diagnosis and assessment of symptomatic lower limb peripheral arterial disease. A Systematic Review. BMJ 2007; 334:1257.

[3] Met R, Bipat S, Legemate DA, Koelemay MJ: Diagnostic Performance of Computed Tomography Angiography in Peripheral Arterial Disease .A Systematic Review and Metaanalysis . JAMA 2009; 301(4):415-424.

[4] Laswed T, Rizzo E, Guntern D, Qanadli SD:Assessment of occlusive arterial disease of abdominal aorta and lower extremities arteries: value of multidetector CT angiography using an adaptive acquisition method Eur Radiol 2008; $18(2): 263-272$.

[5] Gouny P, Verhaeghe A, Nonent M, Gueret G, Badra A: Multi-Detector Row Computed Tomography Angiography : an Alternative Imaging Method for Surgical Strategy in Lower Extremity Arterial Occlusive Disease. ActaChirBelg, 2005; 105:592-601.
[6] Foley WD , Stonely T: CT angiography of the lower extremities. RadiolClin North Am Mar 2010; 48(2):367-96.

[7] Fleischmann D, Hallett RL, Rubin GD: CT angiography of peripheral arterial disease. J VascIntervenRadiol 2006; 17(1): $3-26$.

[8] Rubin GD, Shiau MC, Leung AN, Kee ST, Logan LJ , Sofilos MC: Aorta and iliac arteries: single versus multiple detector-row helical CT angiography. Radiology Jun 2000; 215(3): 670-6.

[9] Ofer A, Nitecki SS, Linn S, Epelman M, Fischer D, Karram T, Litmanovich D, Schwartz H, Hoffman A, Engel A:Multidetector CT angiography of peripheral vascular disease: A prospective comparison with intra-arterial digital subtraction angiography. AJR 2003; 180: 719-724.

[10] Willmann JK, Mayer D, Banyai M: Evaluation of peripheral arterial bypass grafts with multi-detector row CT angiography: comparison with duplex US and digital subtraction angiography. Radiology Nov 2003; 229(2): 46574.

[11] Ozkan U, Oguzkurt L, Tercan F: Atherosclerotic risk factors and segmental distribution in symptomatic peripheral artery disease. J VascIntervRadiol Apr 2009; 20(4):437-41. 